\title{
The Effects of Repetitive Transcranial Magnetic Stimulation at Dorsolateral Prefrontal Cortex in the Treatment of Migraine Comorbid with Depression: A Retrospective Open Study
}

\author{
Saurabh Kumar, Swarndeep Singh, Nand Kumar, Rohit Verma \\ Department of Psychiatry, All India Institute of Medical Sciences, New Delhi, India
}

\begin{abstract}
Objective: The literature on managing migraine non-responsive to pharmacological approaches and that co-occurring with depression is scanty. The comorbid condition predicts a poorer prognosis for migraine as well as depression. The present report assesses efficacy and tolerability of high frequency repetitive transcranial magnetic stimulation (rTMS) over left dorsolateral prefrontal cortex as a treatment modality for migraine with comorbid depression.

Methods: The current retrospective chart review assesses effectiveness of high frequency rTMS over left dorsolateral prefrontal cortex as a treatment modality to manage migraine occurring comorbid with depression in 14 subjects.

Results: The mean scores on Migraine Disability Assessment Test (MIDAS) and depression rating scale reduced significantly from $21.14 \pm 3.01$ and $20.71 \pm 3.95$ at baseline to $13.93 \pm 6.09$ and $14.21 \pm 5.52$ respectively, after rTMS. There was significant improvement in migraine frequency, severity and functional disability assessed using MIDAS scores ( $p$ $<0.05)$ following high frequency rTMS compared to baseline.

Conclusion: There is a role of applying rTMS as a potential therapeutic modality in the integrated management of a distinct subgroup of migraine patients with comorbid depression.
\end{abstract}

KEY WORDS: Transcranial; Brain stimulation; Neuromodulation; rTMS; Migraine; Depression.

\section{INTRODUCTION}

Migraine and depression, as separate disorders are ranked among the top ten leading causes of disability worldwide. ${ }^{1)}$ In several population studies, people with migraine ('migraineurs') were between 2.2 to 4.0 times more likely to suffer from depression than non-migraineurs. ${ }^{2)} \mathrm{A}$ recent population based study comprising of 6,624 participants confirmed that depression is comorbid more than chance with migraine. ${ }^{3)}$ In patients with migraine, comorbidity with depression is associated with greater severity of headache, more disability, higher risk of suicide and predicts a poorer prognosis. ${ }^{4)}$ Conversely, major depressive disorder patients with comorbid mi-

Received: December 15, 2016/Revised: May 18, 2017 Accepted: June 20, 2017

Address for correspondence: Rohit Verma, MD

Department of Psychiatry, All India Institute of Medical

Sciences, Fourth Floor, Teaching Block, Ansari Nagar, New

Delhi, NCT-Delhi 110029, India

Tel: +91-9899461700, Fax: +91-11-26588663

E-mail: drrv321@gmail.com graine have more number of depressive episodes, greater severity of depression, anxiety and somatic symptoms along with poorer health-related quality of life. Furthermore, a bidirectional relationship is reported between migraine and depression, with one disorder increasing the incident risk of the other and vice versa suggesting some common pathogenesis mechanisms (neurobiological or environmental factors) for both the disorders. $^{5,6)}$

Several pharmacological agents for acute and prophylactic treatment of migraine are available. But, still a clinically significant proportion of patients suffer from intractable migraine, with inadequately controlled migraine attacks despite of receiving an adequate trial of multiple prophylactic pharmacotherapies. Thus, there is a need for exploring other novel treatment strategies such as repetitive transcranial magnetic stimulation (rTMS) for treatment of migraine. High frequency rTMS (HF-rTMS) over left dorsolateral prefrontal cortex (Lt-DLPFC) brain area is already an approved treatment option for depression by

(c) This is an Open-Access article distributed under the terms of the Creative Commons Attribution Non-Commercial License (http://creativecommons.org/licenses/by-nc/4.0) which permits unrestricted non-commercial use, distribution, and reproduction in any medium, provided the original work is properly cited. 
the US Food and Drug Administration," with studies showing reversal of hypometabolism/hypoactivity in Lt-DLPFC along with metabolic abnormalities in other brain areas after successful rTMS treatment correlating with its antidepressant effect in patients of depression. ${ }^{8)}$ Prefrontal dysfunction in the form of impaired neuropsychological tests of prefrontal function has been reported in patients with chronic migraine. ${ }^{9)}$ In addition, a study involving healthy volunteers reported reduced capsaicin-induced pain on the dorsum of both the hands on Lt-DLPFC stimulation, with no such effect on right-DLPFC stimulation. ${ }^{10)}$ This suggests that Lt-DLPFC may exert bilateral control over pain, with its stimulation having possible central anti-nociceptive effect. Hence, HF-rTMS over Lt-DLPFC may be used as an integrated therapeutic approach targeting both migraine and comorbid depression simultaneously. Here, we present the retrospective analysis of 14 patients with migraine and comorbid depression who had received treatment with HF-rTMS over Lt-DLPFC assessing its efficacy and safety.

\section{METHODS}

The study reports findings from the retrospective record based review of clinical case files of patients treated at rTMS lab, Department of Psychiatry of a tertiary care treatment center in India between July and October, 2013. Patients of either gender, above the age of 18 years with a diagnosis of migraine without aura according to the International Headache Society criteria, ${ }^{11)}$ with failure to respond to a trial of at least two migraine prophylactic medications at adequate dose and duration (at least 12 weeks) and a diagnosis of comorbid unipolar depression, with a score of more than 14 on Hamilton Depression Rating Scale (HAM-D) ${ }^{12)}$ and failure to respond to at least one antidepressant trial at adequate dose and duration were included in the present study. Patients with any other psychiatric comorbidity (based on detailed clinical interview by a psychiatrist), history of seizures, neurosurgical metallic implant, cardiac pacemaker or inner ear prosthesis, pregnancy or unstable medical condition were excluded.

HF-rTMS in accordance with the updated safety guidelines was given, ${ }^{13)}$ after taking written informed consent from participants as a routine part of treatment at rTMS lab. Magstim Rapid ${ }^{2}$ device (Magstim, Whiteland, UK) with a 70-mm figure-of-eight air-film coil was used to administer rTMS. The resting motor evoked potential (MEP) was determined using an electromyogram, recording from the right-sided abductor pollicis brevis in accordance with the International Federation of Clinical Neurophysiology recommendations. ${ }^{13)}$ The resting motor threshold (RMT) was defined as the minimum stimulus intensity that produced a MEP (about $50 \mu \mathrm{V}$ in five out of 10 trials) at rest. The coil was placed over the Lt-DLPFC, as per the standard procedure, determined by moving the TMS coil $5.0 \mathrm{~cm}$ rostrally from the right abductor pollicis brevis motor threshold area, along a left superior oblique plane with a rotation point about the tip of the patient's nose. $^{14)}$ The stimulation parameters used were $20-\mathrm{Hz}$ frequency, stimulation intensity at $110 \%$ of RMT, 5-seconds train duration, inter-train interval of 20 seconds and 10 trains per session. Each session of rTMS consisted of 1,000 pulses/day delivered in 250 seconds. A total of 15 sessions of rTMS, five days per week (from Monday to Friday) over a period of three weeks were given. The medical treatment of the patients was unchanged for at least 12 weeks prior-to and during the entire study period.

All patients were assessed in detail prior to starting of treatment at the rTMS lab and they were asked to maintain a self-rated headache diary in which the frequency of headache (number of days) and severity of headache rated on a four point Likert scale ( $0=$ no pain, $1=$ mild pain, $2=$ moderate pain, and $3=$ severe pain) was recorded. Migraine Disability Assessment Test (MIDAS) was used to assess the functional disability due to migraine attacks in the past one month and HAM-D rating scale was applied by a psychiatrist to rate the depressive symptoms. MIDAS is the most widely used measure of disability in headache research. ${ }^{15)}$ It consists of five questions inquiring about the number of days during which headache presence disrupted (partially or totally) paid and school work, household work, and leisure/family/social duties. Evaluation was done at the baseline (T0), after completion of rTMS treatment (T1), and subsequently at four and eight weeks interval (T2 and T3) after treatment completion.

The study was approved by the institute ethics committee of All India Institute of Medical Sciences (IEC-133/04. 03.2016, RP-8/2016).

The various outcome measures at different time intervals were evaluated by one-way repeated measures analysis of variance. Simple effect analysis using paired $t$ test 
Table 1. Assessment over repetitive transcranial magnetic stimulation treatment course and follow up

\begin{tabular}{lcccrrrr}
\hline \multirow{2}{*}{ Assessment } & \multirow{2}{*}{$\begin{array}{c}\text { Baseline } \\
\text { (T0) }\end{array}$} & $\begin{array}{c}\text { After 15 sessions } \\
\text { (T1) }\end{array}$ & \multirow{2}{*}{$\begin{array}{c}\text { After 4 weeks } \\
\text { (T2) }\end{array}$} & $\begin{array}{c}\text { After 8 weeks } \\
\text { (T3) }\end{array}$ & & \multicolumn{3}{c}{$p$ value } \\
\cline { 6 - 8 } & & & & & Pair 1 & Pair 2* & Pair 3* \\
\hline HAM-D & $20.71 \pm 3.95$ & $14.21 \pm 5.52$ & $13.79 \pm 6.35$ & $13.79 \pm 6.10$ & $<0.001$ & $<0.001$ & $<0.001$ \\
MIDAS & $21.14 \pm 3.01$ & $13.93 \pm 6.09$ & $14.01 \pm 6.38$ & $14.07 \pm 5.75$ & $<0.001$ & $<0.001$ & $<0.001$ \\
Headache frequency & $2.86 \pm 0.66$ & $1.93 \pm 1.14$ & $1.93 \pm 1.14$ & $1.93 \pm 0.82$ & 0.006 & 0.006 & 0.001 \\
Headache severity & $2.43 \pm 0.51$ & $1.50 \pm 0.65$ & $1.43 \pm 0.51$ & $1.43 \pm 0.51$ & $<0.001$ & $<0.001$ & $<0.001$ \\
\hline
\end{tabular}

Values are presented as mean \pm standard deviation.

HAM-D, Hamilton Depression Rating Scale; MIDAS, Migraine Disability Assessment Test.

Pair 1, baseline vs. after 15 sessions; Pair 2, baseline vs. after 4 weeks; Pair 3, baseline vs. after 8 weeks; ${ }^{*} p$ value for paired $t$ test.

was applied between pair-1 (T0 and T1), pair-2 (T0 and $\mathrm{T} 2$ ) and pair-3 (T0 and T3) to compare the difference between outcome measures at different time points as compared to baseline (Table 1), when overall effect was found to be significant in one-way repeated measures analysis of variance. Pearson correlation analysis was used to examine whether changes in scores of migraine disability and depression rating scales correlate. The difference was considered significant, if the $p$ value was $<0.05$. The statistical analysis was done using IBM SPSS Statistics version 21.0 (IBM Co., Armonk, NY, USA).

\section{RESULTS}

Among the study population, $11(78.6 \%)$ participants were males and $3(21.4 \%)$ belonged to female gender. All the participants were right handed with the mean age of $40.57 \pm 7.46$ years (range, 28-55 years). The mean duration of migraine for the study population at the time of rTMS initiation was $13.71 \pm 6.17$ years. The mean number of failed trials of prophylactic medication reported was $2.21 \pm 0.42$. There were no serious adverse effects in any patient during or after rTMS and it was well tolerated by all the patients. Repeated measures analysis of variance showed significant changes in MIDAS scores (degree of freedom $[\mathrm{dF}]=3, \mathrm{~F}=18.11, p<0.001)$, HAM-D scores $(\mathrm{dF}=3, \mathrm{~F}=29.19, p<0.001)$, frequency $(\mathrm{dF}=3, \mathrm{~F}=10.63, p$ $<0.001)$ and severity $(\mathrm{dF}=3, \mathrm{~F}=25.15, p<0.001)$ of migraine attacks with time. There was significant improvement in migraine frequency, severity and functional disability assessed using MIDAS scores $(p<0.05)$ following rTMS compared to baseline (Table 1). Further, the mean HAM-D scores improved after rTMS as compared to baseline, with more than $50 \%$ reduction in HAM-D scores (i.e., response to treatment in depression) in five out of 14

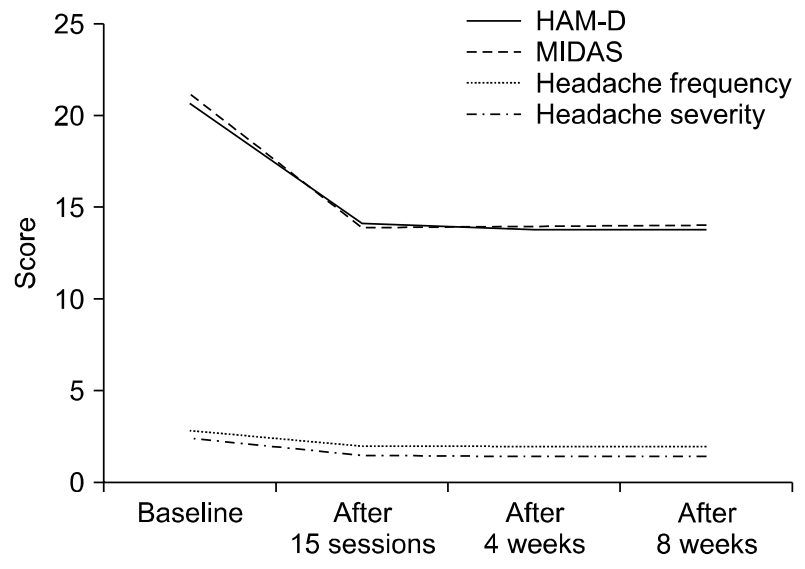

Fig. 1. Depiction of change in scores over the assessment period. HAM-D, Hamilton Depression Rating Scale; MIDAS, Migraine Disability Assessment Test.

participants $(35.7 \%)$. This improvement sustained to significant level as compared to baseline over the 8 weeks follow-up period (Fig. 1). The changes in MIDAS and HAM-D scores after 15 sessions of rTMS did not significantly correlate with each other $(r=0.354, n=14$, $p=0.214$ ).

\section{DISCUSSION}

The result of our study showed that HF-rTMS over Lt-DLPFC significantly reduced the headache frequency, severity and functional disability at the end of treatment as compared to baseline. Moreover, the improvement was sustained over the next eight weeks follow-up period suggesting that the response was less likely to be due non-specific factors or a placebo effect. This is similar to the results reported in a randomized sham-controlled trial previously; involving 11 patients of chronic migraine with 12 sessions of $20-\mathrm{Hz}$ rTMS over Lt-DLPFC given in the ac- 
tive group, resulting in significant decrease in headache frequency and severity as compared to sham-treated group. ${ }^{16)}$

The exact biological mechanism through which HF-rTMS over Lt-DLPFC exerts its beneficial effects in migraine is not clearly established. The Lt-DLPFC stimulation might exert an inhibitory effect on pain perception by activation of supra-spinal pathways or by resetting or reducing the fronto-limbic dysfunction seen in chronic painful conditions, particularly chronic migraine. ${ }^{17,18)}$ Further, HF-rTMS over frontal cortex has been reported to increase the basal low plasma $\beta$ endorphin levels in migraine patients, which was associated with improvement of migraine. $^{19)}$

However, a recent randomized, double-blind, shamcontrolled trial involving 18 patients of migraine with 23 sessions of $10-\mathrm{Hz}$ rTMS over Lt-DLPFC given in the active group, failed to show any beneficial effect of rTMS over sham treatment. ${ }^{20)}$ This disagreement in the results of the above study may be due to differences in the stimulation parameters (10-Hz vs. $20-\mathrm{Hz}$ ) and site of stimulation due to use of different methods for localisation of Lt-DLPFC area. Further, it may be due to differential treatment response in various subgroups of migraine, with Hf-rTMS over Lt-DLPFC being particularly effective in migraine with comorbid depression patients. This is indeed supported by the findings of a recent magnetic resonance based neuroimaging study involving 4,269 participants reporting that comorbid migraine and depression was associated with smaller total brain volume than those with none or either one of these conditions and may represent a distinct clinical phenotype with different long-term sequelae. $^{21)}$

In the present study, significant reduction of mean HAM-D scores was seen at the end of treatment as compared to baseline. It is interesting to note here that the change in disability due to migraine wasn't significantly correlated with change in depression severity, possibly indicating effect of rTMS on migraine symptoms independent of its effect on depressive symptoms. However, the mean HAM-D score was still above 14 at the end of treatment. This could be because of the lack of sufficient specificity of various questionnaires such as HAM-D used for the assessment of depression in headache patients. ${ }^{22)}$ It may be sometimes difficult for the patients to differentiate changes such as decreased energy, sleep disturbances, weight changes, difficulties in concentration and some other somatic symptoms occurring secondary to headaches from that due to a primary mood disorder. Hence, instruments such as Beck Depression Inventory-II which gives greater weightage to depressive cognitions may be a better instrument for assessing depression in migraine patients in future studies. Further, the decreased effect of rTMS on depression in the present study in contrast to that reported in several previous studies, ${ }^{23)}$ may be due to the differences in type of patients (moderate depression vs. severe depression), stimulation parameters (20-z vs. $10-\mathrm{Hz})$, number of sessions (15 vs. 20), number of pulses delivered (1,000 pulses/day vs. 3,000 pulses/day) or different methods of determining Lt-DLPFC used. The small sample size (14 patients) of the current study may not be adequately powered statistically to detect the effect of rTMS on depression.

The present study is a retrospective analysis of an open-label trial of HF-rTMS given to patients of migraine with comorbid depression with no control group, is prone to biases inherent in such design limiting its generalization. In a meta-analysis to evaluate the placebo response rate in published clinical trials, the pooled estimate of placebo response in migraine prophylaxis was reported around $21 \%{ }^{24)}$ The response rate for migraine symptoms in the present study was much higher (about 50\%) and was maintained over at least two-months follow-up period, suggesting placebo effect to be less likely. However, placebo effects cannot be definitively ruled out because of absence of randomization and control group in the study. Further, repeated measures analysis of variance with depression as a covariate (ANCOVA) was not possible in present study because scores on depression rating scale also showed significant reduction over time with rTMS treatment and the assumption that the covariate is independent of treatment effects, required for conducting ANCOVA was not satisfied.

The present study suggests use of HF-rTMS over Lt-DLPFC as a potential therapeutic modality having beneficial effects in both migraine prophylaxis and amelioration of depression, which may be used in the integrated management of a distinct subgroup of migraine patients with comorbid depression. Further, large-scale, double-blind, placebo-controlled trials are needed to better characterize the stimulation patterns and confirm the therapeutic role of HF-rTMS over Lt-DLPFC in migraine 
with comorbid depression.

\section{Acknowledgments}

The authors thank Mr. Vikas Kumar and Ms. Komal Gupta for constantly providing support for the functioning of rTMS machine.

\section{REFERENCES}

1. Global Burden of Disease Study 2013 Collaborators. Global, regional, and national incidence, prevalence, and years lived with disability for 301 acute and chronic diseases and injuries in 188 countries, 1990-2013: a systematic analysis for the Global Burden of Disease Study 2013. Lancet 2015;386: 743-800.

2. Hamelsky SW, Lipton RB. Psychiatric comorbidity of migraine. Headache 2006;46:1327-1333.

3. Lampl C, Thomas H, Tassorelli C, Katsarava Z, Laínez JM, Lantéri-Minet M, et al. Headache, depression and anxiety: associations in the Eurolight project. I Headache Pain 2016; $17: 59$.

4. Lantéri-Minet M, Radat F, Chautard MH, Lucas C. Anxiety and depression associated with migraine: influence on migraine subjects' disability and quality of life, and acute migraine management. Pain 2005;118:319-326.

5. Breslau N, Lipton RB, Stewart WF, Schultz LR, Welch KM. Comorbidity of migraine and depression: investigating potential etiology and prognosis. Neurology 2003;60:1308-1312.

6. Baskin SM, Smitherman TA. Migraine and psychiatric disorders: comorbidities, mechanisms, and clinical applications. Neurol Sci 2009;30 Suppl 1:S61-S65.

7. Aleman A. Use of repetitive transcranial magnetic stimulation for treatment in psychiatry. Clin Psychopharmacol Neurosci 2013;11:53-59.

8. Li CT, Wang SJ, Hirvonen J, Hsieh JC, Bai YM, Hong CJ, et al. Antidepressant mechanism of add-on repetitive transcranial magnetic stimulation in medication-resistant depression using cerebral g/ucose metabolism. J Affect Disord 2010;127: 219-229.

9. Mongini F, Keller R, Deregibus A, Barbalonga E, Mongini T. Frontal lobe dysfunction in patients with chronic migraine: a clinical-neuropsychological study. Psychiatry Res 2005;133: 101-106.

10. Brighina F, Cosentino G, Vigneri S, Talamanca S, Palermo A, Giglia G, et al. Abnormal facilitatory mechanisms in motor cortex of migraine with aura. Eur J Pain 2011;15:928-935.

11. Headache Classification Subcommittee of the International
Headache Society. The International Classification of Headache Disorders: 2nd edition. Cephalalgia 2004;24 Supp/ 1:9-160.

12. Hamilton M. A rating scale for depression. J Neurol Neurosurg Psychiatry 1960;23:56-62.

13. Rossi S, Hallett M, Rossini PM, Pascual-Leone A; Safety of TMS Consensus Group. Safety, ethical considerations, and application guidelines for the use of transcranial magnetic stimulation in clinical practice and research. Clin Neurophysiol 2009;120:2008-2039.

14. Pascual-Leone A, Rubio B, Pallardó F, Catalá MD. Rapid-rate transcranial magnetic stimulation of left dorsolateral prefrontal cortex in drug-resistant depression. Lancet 1996;348: 233-237.

15. Stewart WF, Lipton RB, Whyte J, Dowson A, Kolodner K, Liberman JN, et al. An international study to assess reliability of the Migraine Disability Assessment (MIDAS) score. Neurology 1999;53:988-994.

16. Brighina F, Piazza A, Vitello G, Aloisio A, Palermo A, Daniele $\mathrm{O}$, et al. rTMS of the prefrontal cortex in the treatment of chronic migraine: a pilot study. J Neurol Sci 2004;227:67-71.

17. Lorenz J, Minoshima S, Casey KL. Keeping pain out of mind: the role of the dorsolateral prefrontal cortex in pain modulation. Brain 2003;126:1079-1091.

18. Rome JD. Limbically augmented pain syndromes: kindling andcorticolimbic sensitisation. Cephalalgia 2001;21:241245. T1-1.

19. Misra UK, Kalita J, Tripathi GM, Bhoi SK. Is $\beta$ endorphin related to migraine headache and its relief? Cephalalgia 2013; 33:316-322

20. Conforto AB, Amaro $E$ Jr, Gonçalves AL, Mercante JP, Guendler VZ, Ferreira JR, et al. Randomized, proof-of-principle clinical trial of active transcranial magnetic stimulation in chronic migraine. Cephalalgia 2014;34:464-472.

21. Gudmundsson LS, Scher AI, Sigurdsson S, Geerlings MI, Vidal JS, Eiriksdottir G, et al. Migraine, depression, and brain volume: the AGES-Reykjavik Study. Neurology 2013;80:21382144.

22. Maizels M, Smitherman TA, Penzien DB. A review of screening tools for psychiatric comorbidity in headache patients. Headache 2006:46 Suppl 3:598-S109.

23. Perera T, George MS, Grammer G, Janicak PG, Pascual-Leone A, Wirecki TS. The Clinical TMS Society consensus review and treatment recommendations for TMS therapy for major depressive disorder. Brain Stimul 2016;9:336-346.

24. Macedo A, Baños JE, Farré M. Placebo response in the prophylaxis of migraine: a meta-analysis. Eur J Pain 2008;12:68-75. 